\title{
The performance of drag models on flow behaviour in the CFD simulation of a fluidized bed
}

\author{
B. M. Halvorsen ${ }^{1}$, J. P. du Plessis ${ }^{2} \&$ S. Woudberg ${ }^{2}$ \\ ${ }^{I}$ Department of Process Technology, Telemark University College, \\ Porsgrunn, Norway \\ ${ }^{2}$ Department of Applied Mathematics, \\ University of Stellenbosch, South Africa
}

\begin{abstract}
The aim of this study is to verify the use of a newly developed drag model in the simulation of fluidized beds. The drag model is based on a geometric description of the geometry found in a fluidised bed, treating it as a spatially and temporally variable inhomogeneous, locally isotropic, porous medium. Account is taken of the fact that flow conditions in low porosity parts of a bed can be viewed as flow between particles. At high porosities the bed resembles flow past the particles of a dilute assemblage and for that the current model is complemented with results from other models. The new drag model, as well as other models found in literature, was tested in the numerical simulations. Computational results are compared mutually, as well as to experimental data, and the differences and discrepancies discussed.
\end{abstract}

Keywords: fluidized bed, numerical simulation, drag model.

\section{Introduction}

Fluidized beds are widely used in industrial chemical processes. In a fluidized bed gas is passing upwards through a bed of particles and the earliest applications of fluidization were for the purpose of enhancing chemical reactions. Fluidized beds in chemical industry include two main types of reactions, catalytic gas phase reactions and gas-solid reactions. In catalytic gas phase reactions the particles are not undergoing any chemical reaction. This is 
the principal of oil cracking for manufacturing of various chemical substances. In gas-solid reactions the fluidized particles are involved in the reactions and undergo a phase change. An example of this type of process is combustion or gasification of coal. Other application of fluidized beds are drying and coating of solids.

Fluidized beds are applied in industry due to their large contact area between phases, which enhances chemical reactions, heat transfer and mass transfer. The efficiency of fluidized beds is highly dependent of flow behaviour and knowledge about flow behaviour is essentially for scaling, design and optimisation. Computational fluid dynamics (CFD) has during the last decades become a useful tool in predicting flow behaviour in fluidized bed processes. However, further model development and verification of the model and the numerical procedure are still needed.

Gravity and drag are the most predominant terms in the solid phase momentum equation and the application of different drag models has significant impact on the flow of the solid phase by differently influencing the predicted bed expansion and the solid concentration in the dense phase regions of the bed. Yasuna et al [1], Halvorsen and Mathiesen [2], Ibsen [3] and Bokkers et al [4] showed that the solution of their models is sensitive to the drag coefficient. In general, the performance of most current models depends on the accuracy of the drag formulation.

The Ergun Equation [5], Bird et al [6] is frequently used as drag model for calculating pressure gradients during flow in a fluidized beds. An updated value for the first coefficient in the Ergun equation from 150 to 180 was reported by MacDonald et al [7] and may also be used as an improved empirical model. Both were, however, derived empirically for Newtonian flow through packed beds in a fairly narrow band of porosities around $40 \%$ and their generalization to more general physical situations cannot be performed, but in an approximate and empirical manner. In active fluidized beds the void fraction can change over the full range from zero through unity and the model used in numerical simulations should be equally versatile.

The Ergun equation may be written as follows [5]:

$$
\mathrm{FD}^{2}=\frac{\mathrm{A}(1-\varepsilon)^{2}}{\varepsilon^{3}}+\frac{\mathrm{B}(1-\varepsilon)}{\varepsilon^{3}} \mathrm{Re}=\frac{150(1-\varepsilon)^{2}}{\varepsilon^{3}}+\frac{1.75(1-\varepsilon)}{\varepsilon^{3}} \mathrm{Re}
$$

where $\varepsilon$ is the porosity and Re the local Reynolds number expressed by:

$$
\operatorname{Re}=\frac{\rho_{\mathrm{g}} \mathrm{qD}}{\mu_{\mathrm{g}}}
$$

and $\mathrm{q}$ is the superficial velocity:

$$
q=\varepsilon\left|U_{g}-U_{s}\right|
$$

Here $U_{g}$ and $U_{s}$ are the local gas and solid velocities respectively.

Gidaspow [8] combined the Ergun equation with the equations of Rowe [9] and Wen and $\mathrm{Yu}[10]$ to get a drag model that can cover the whole range of porosities. The following equation of Wen and $\mathrm{Yu}$ is used for a voidage above 0.80 : 


$$
\mathrm{FD}^{2}=\frac{3}{4} \mathrm{C}_{\mathrm{D}} \frac{\varepsilon_{\mathrm{s}} \varepsilon \cdot \mathrm{D}}{\mu} \rho_{\mathrm{g}}\left|U_{\mathrm{g}}-U_{\mathrm{s}}\right| \varepsilon^{-2.65}, \text { for } \varepsilon>0.8
$$

Rowe [9] related the friction coefficient, $\mathrm{C}_{\mathrm{D}}$, to Reynolds number by:

$$
\begin{array}{ll}
C_{D}=\frac{24}{\operatorname{Re}_{\mathrm{s}}}\left(1+0.15 \mathrm{Re}^{0.687}\right), & \operatorname{Re}_{\mathrm{s}} \leq 1000 \\
\mathrm{C}_{\mathrm{D}}=0.44, & \operatorname{Re}_{\mathrm{s}}>1000
\end{array}
$$

The particle Reynolds number, $\mathrm{Re}_{\mathrm{s}}$, is expressed by:

$$
\operatorname{Re}_{\mathrm{s}}=\frac{\mathrm{D} \rho_{\mathrm{g}}\left|U_{\mathrm{g}}-U_{\mathrm{s}}\right| \varepsilon_{\mathrm{s}}}{\mu_{\mathrm{g}}}
$$

The MacDonald drag model is given, for the entire range of porosities, by:

$$
F D^{2}=\frac{180(1-\varepsilon)^{2}}{\varepsilon^{3}}+\frac{1.8(1-\varepsilon)}{\varepsilon^{3}} \mathrm{Re}
$$

Gibilaro et al [11] proposed the following drag model:

$$
F D^{2}=\frac{3}{4} \mathrm{C}_{\mathrm{D}} \frac{\varepsilon_{\mathrm{s}} \varepsilon \cdot \mathrm{D}}{\mu} \rho_{\mathrm{g}}\left|U_{\mathrm{g}}-U_{\mathrm{s}}\right|
$$

where the friction coefficient, $\mathrm{C}_{\mathrm{D}}$, is expressed by:

$$
\mathrm{C}_{\mathrm{D}}=\frac{4}{3}\left(\frac{17.3}{\mathrm{Re}_{\mathrm{s}}}+0.336\right) \varepsilon^{-2.80}
$$

\section{The proposed drag model}

The deterministic model presented here is based on a fixed simplistic geometrical layout but the voidage can take any value according to the properties found within any part of the bed Du Plessis [12]. Flow conditions are then assumed according to the geometry of the flow passages, the void fraction and the interstitial Reynolds number. In this manner elaboration towards more complex behaviour can be performed in a systematic manner and discrepancies and unexpected behavioural characteristics can be analysed in a scientific manner. The local Reynolds number at any point in the bed is defined as above. The Reynolds number is taken into account when determining the local drag coefficient.

\subsection{Creep flow solution at low Reynolds number flow}

The low voidage drag model of Du Plessis was improved for creep flow relative to an isotropic granular material, Woudberg et al [13]. Since it is impossible to envisage an isotropic geometric arrangement of particles it is assumed that the properties of an isotropic medium are resembled by the average of the properties of flow in three perpendicular directions though an arrangement where the particles are maximally (fully) staggered in one direction and non-staggered in the other two directions. In the one direction the flow thus experiences a fully 
staggered configuration with maximal tortuosity of the streamlines. In the other two directions the flow lines are straight and stagnant regions are formed between streamwise adjacent solid particles.

At high voidages creep flow through an assemblage of particles is more appropriately considered as flow past each of the particles than as flow between solid constituents. A model proposed by Hasimoto [14], for flow past a particle that is imbedded in an assemblage of other particles is therefore used in the present work to describe the creep flow situation at high voidage factors. It may also be noted that there is no large difference between the flow conditions in regular or staggered arrays when the voidage is high.

An asymptotic matching technique Churchill and Usagi [15] is used to combine the two asymptotic solutions into one equation, facilitating their use in numerical simulations. This combined equation for all voidage factors is then considered as an asymptotic condition for creep flow situations in the bed.

\subsection{Inertial flow solution at intermediate Reynolds number flow}

In regions where the Reynolds number is well above unity, local areas of recirculation develop at the lee side of particles, giving rise to inertial effects in the so-called Forchheimer regime. These effects can be modelled as momentum effects resulting in a pressure drop over each particle. This is not yet in the turbulence regime but, since numerous experiments (e.g. MacDonald et al) suggest a fairly established asymptotic behaviour, these laminar conditions will be considered as adhering to an asymptotic law at intermediate Reynolds number values of flow locally within in the bed.

\subsection{Asymptote matching}

The asymptote matching technique is again applied to match two asymptotic solutions, namely that of creep flow and that of inertial but still laminar flow Woudberg [16], resulting in equation (10), where $\mathrm{F}$ is the drag factor (inverse of the permeability) and D the average grain diameter. Here the first and last terms respectively reflect the creep solutions at low and high voidage and the middle term the inertial flow conditions when interstitial recirculation occurs.

$$
\begin{gathered}
F D^{2}=\frac{26.8(1-\varepsilon)^{4 / 3}}{\left(1-(1-\varepsilon)^{1 / 3}\right)\left(1-(1-\varepsilon)^{2 / 3}\right)^{2}}+\frac{(1-\varepsilon)}{\varepsilon\left(1-(1-\varepsilon)^{2 / 3}\right)^{2}} \operatorname{Re} \\
+\left(162 \pi^{2}\right)^{1 / 3}(1-\varepsilon)\left(1+1.79(1-\varepsilon)^{1 / 3}\right)
\end{gathered}
$$

If the present solution is compared to the existing models, the coefficients $A$ and $\mathrm{B}$ of the Ergun equation are functions of the void fraction, respectively as follows:

$$
A=\frac{26.8 \varepsilon^{3}}{(1-\varepsilon)^{2 / 3}\left(1-(1-\varepsilon)^{1 / 3}\right)\left(1-(1-\varepsilon)^{2 / 3}\right)^{2}}
$$


and

$$
B=\frac{\varepsilon^{2}}{\left(1-(1-\varepsilon)^{2 / 3}\right)^{2}}
$$

It is interesting to note that the new model yields an effective B-value that decreases almost linearly from 2.25 at zero voidage to unity at total voidage. Conversely the coefficient $\mathrm{A}$ is predicted as 185 at very low voidage, increasing steadily to about 785 at a voidage factor of unity. Differences in magnitudes among the models are thus evident and this should reflect in the drag profiles predicted.

\section{Physical description of bed dynamics}

Computational and experimental studies have been performed on a 2-D fluidized bed with a central jet. The advantage of using a bed with a jet is that the jet establishes the flow pattern, and this problem is easier to model than uniform fluidization. In the experimental studies a digital video camera was used to measure bubble sizes and bubble velocities.

Spherical glass particles with a mean diameter of $490 \mu \mathrm{m}$ are used in the experiments and the simulations. For these particles the inter-particle forces are negligible and bubbles are formed as the gas velocity reaches the minimum fluidization velocity, Geldart [17]. The bed expansion is small compared to other types of particles. Small bubbles are formed close to the air distributor and the bubble size increase with distance above the distributor. The bubble size also increases with the excess gas velocity which is defined as the difference between the gas velocity and the minimum fluidization velocity, Geldart [17]. Coalescence is the predominant phenomenon of this group of powders and the bubble size is roughly independent of mean particle size. Most bubbles rise faster than the interstitial gas velocity.

\section{Computational procedure}

The computational work is performed by using the CFD model (FLOTRACSMP-3D). The CFD code is based on a multi-fluid Eulerian description of the phases. The kinetic theory for granular flow forms the basis for the turbulence modelling of the solid phase. The CFD code was proposed by Mathiesen et al [18] and modified by Halvorsen [19] to improve its use in dense particle systems like bubbling fluidized beds. At high solid volume fraction, sustained contacts between particles occur and the resulting frictional stresses must be accounted for in the description of the solid phase stress.

FLOTRACS-MP-3D is a gas/solid flow model, which is generalized for one gas phase and $\mathrm{N}$ number of solid phases. The gas phase turbulence is modelled by a sub-grid scale (SGS) model proposed by Deardorff [20]. The largest scales are simulated directly, whereas the small scales are modelled with the SGS turbulence model. In order to model the fluctuations in the solid phases a 
conservation equation for granular temperature is solved for each solid phase. The governing equations given are solved by a finite volume method, where the calculation domain is divided into a finite number of non-overlapping control volumes. The simulations are performed using two-dimensional Cartesian coordinates.

The conservation equations are integrated in space and time. This integration is performed using second order upwind differencing in space and is fully implicit in time. The set of algebraic equations is solved by a tri-diagonal matrix algorithm (TDMA), except for the volume fraction where a point iteration method is used. Partial elimination algorithm (PEA) generalized to multiple phases is used to decouple the drag forces. The inter-phase slip algorithm (IPSA) is used to take care of the coupling between the continuity and the velocity equations.

\subsection{Computational set-up and results}

A two-dimensional Cartesian co-ordinate system is used to describe the geometry. The grid is uniform in both horizontal and vertical direction. Computational set-up for glass particles is given in Table 1. Simulations have been run with one solid phase of identical particles, all of the same size.

Table 1: $\quad$ Computational set-up and conditions, glass particles.

\begin{tabular}{|c|c|c|c|}
\hline \multicolumn{2}{|l|}{ Design: } & \multicolumn{2}{|l|}{ Grid: } \\
\hline Height & $63.0 \mathrm{~cm}$ & Horizontal grid size & $5.0 \mathrm{~mm}$ \\
\hline Width & $19.5 \mathrm{~cm}$ & Vertical grid size & $10.0 \mathrm{~mm}$ \\
\hline Initial bed height & $33.6 \mathrm{~cm}$ & & \\
\hline Initial voidage & 0.50 & & \\
\hline & & Flow specifications: & \\
\hline \multirow{3}{*}{ Mean diameter } & $490 \mu \mathrm{m}$ & Jet velocity & $4.90 \mathrm{~m} / \mathrm{s}$ \\
\hline & & Fluidization velocity & $0.29 \mathrm{~m} / \mathrm{s}$ \\
\hline & & $\begin{array}{l}\text { Maximum volume } \\
\text { fraction of solids }\end{array}$ & 0.64356 \\
\hline
\end{tabular}

Simulations are performed with the drag models of Du Plessis and Woudberg, Ergun/Wen and Yu, MacDonald et al and Gibilaro et al as described above. Figure 1 shows a comparison of the experimental and computational bubbles at time $320 \mathrm{~ms}$. It can be seen that the simulations with the models of Du Plessis, Ergun/Wen and $\mathrm{Yu}$ and MacDonald give very good agreement with the experiment according to bubble velocity. These models also give a symmetric bubble. Further comparison of these three models with the experimental result show that the Ergun model gives the most realistic bubble size and bed expansion. The Du Plessis model gives a slightly larger bubble and a higher bed expansion than the MacDonald model. The Gibilaro model gives an unsymmetrical first bubble and the bubble velocity differs significantly from the experimental bubble velocity. The bed expansion is too low and unphysical high solid fractions are observed. 

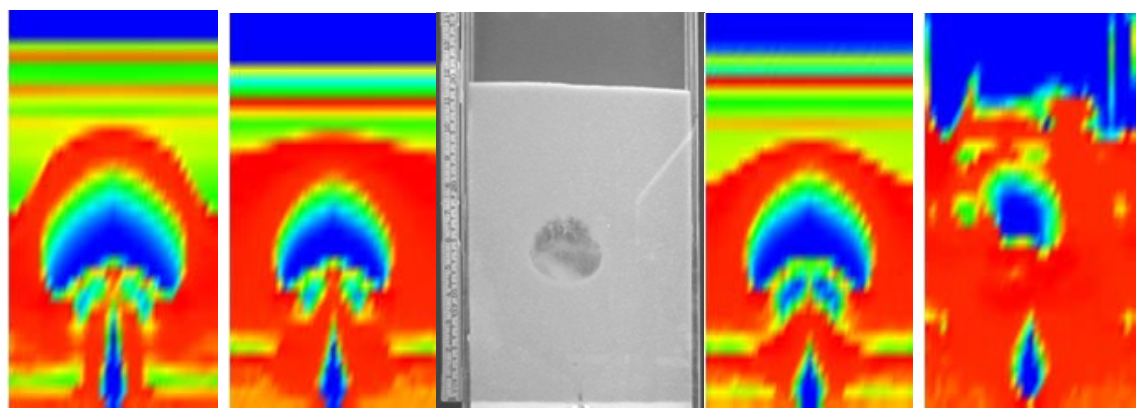

$\mathrm{t}=320 \mathrm{~ms} \quad \mathrm{t}=320 \mathrm{~ms}$

$$
\mathrm{t}=320 \mathrm{~ms}
$$

$\mathrm{t}=320 \mathrm{~ms}$

$\mathrm{t}=320 \mathrm{~ms}$

(Du Plessis) (Ergun,Wen and Yu) (experimental) (MacDonald) (Gibilaro)

Figure 1: $\quad$ Computational vs. experimental bubble at time $320 \mathrm{~ms}$.
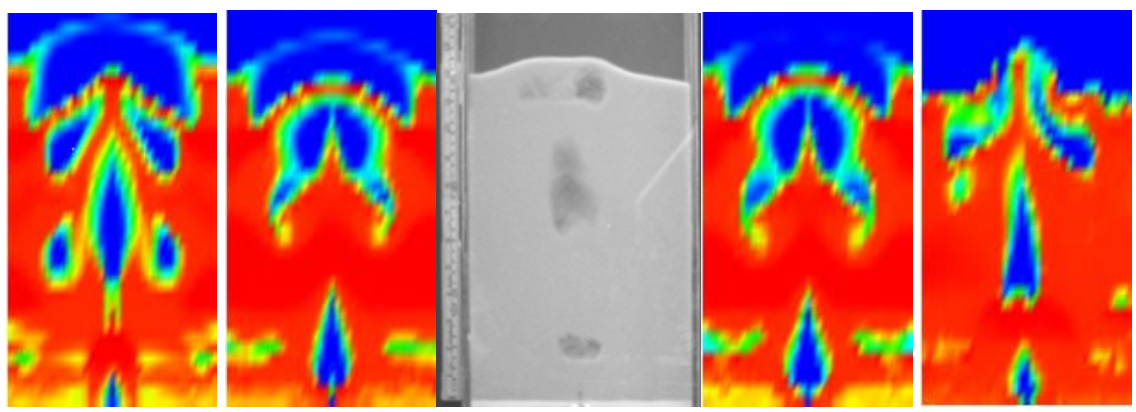

$\mathrm{t}=620 \mathrm{~ms} \quad \mathrm{t}=600 \mathrm{~ms}$

$\mathrm{t}=740 \mathrm{~ms}$

$\mathrm{t}=620 \mathrm{~ms}$

$\mathrm{t}=540 \mathrm{~ms}$

(Du Plessis) (Ergun, Wen and Yu) (experimental) (MacDonald) (Gibilaro)

Figure 2: $\quad$ Computational vs. experimental bubble near the top of the bed.

Figure 2 shows a comparison of the computational and experimental result at the time when the first bubbles erupt. Also at this level the Gibilaro model differ considerably from the others. For all the models the time between bubble creation and bubble eruption is shorter than for the experimental bubble. It can also be seen that all the models give continuous bubble formation. The Ergun and the MacDonald models give about the same shape and velocity for the second bubble. The Du Plessis and the Gibilaro models give the best agreement with the subsequent experimental bubble according to shape and size.

The two figures above show that the computational bubble behaviour is influenced significantly by the particular drag model used. Figures 3 and 4 show the computational drag $\left(\mathrm{FD}^{2}\right)$ as a function of radial position in the bed at height $0.2 \mathrm{~m}$ and $0.3 \mathrm{~m}$ respectively. The drag is averaged over a time lapse of $800 \mathrm{~ms}$. It can be seen from Figure 3 that the drag is low in the centre of the bed where the bubbles are located, and the drag increases towards the walls where the particle concentration is high. At height $0.2 \mathrm{~m}$ Du Plessis, Ergun and MacDonald 
predict about the same drag in the centre of the bed. There are some discrepancies between the models towards the walls. The Gibilaro model gives a lower drag than the other models in all radial positions at this height.

At a height $0.3 \mathrm{~m}$ in the bed, the bubbles erupt or are about to erupt. This can be seen from the drag profile shown in Figure 4. Du Plessis, Ergun and MacDonald predict about the same drag profiles. The drag is rather low in all positions, but some peaks with higher drag are observed in the centre and in a middle core, which indicates a higher particle concentration in these areas. Also at height $0.3 \mathrm{~m}$ Gibilaro's model differ significantly from the others. Gibilaro's model gives a drag close to zero which indicates that there are almost no particles at this height.

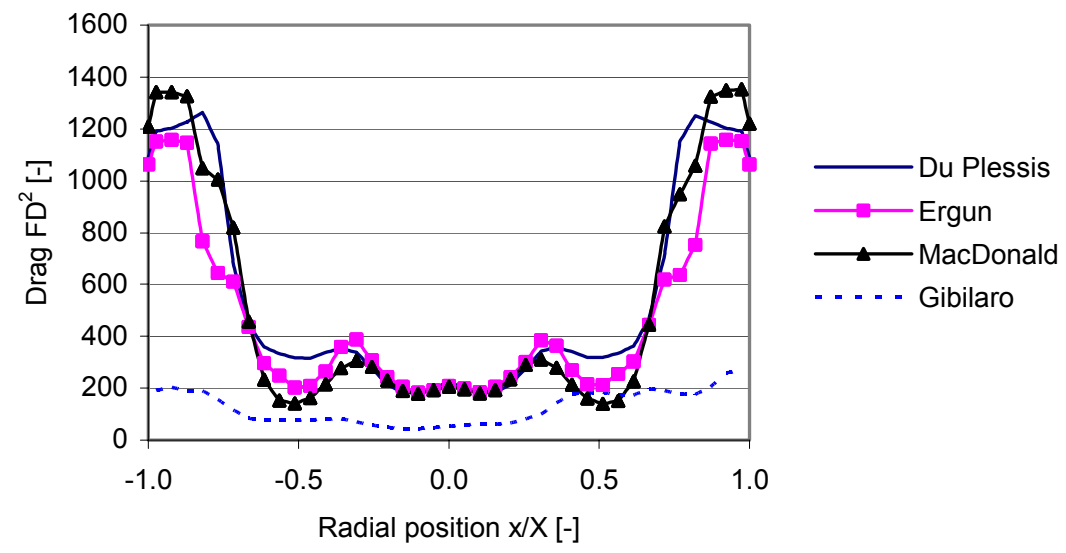

Figure 3: Drag $\left(\mathrm{FD}^{2}\right)$ as a function of radial position at height $0.2 \mathrm{~m}$ in the bed.

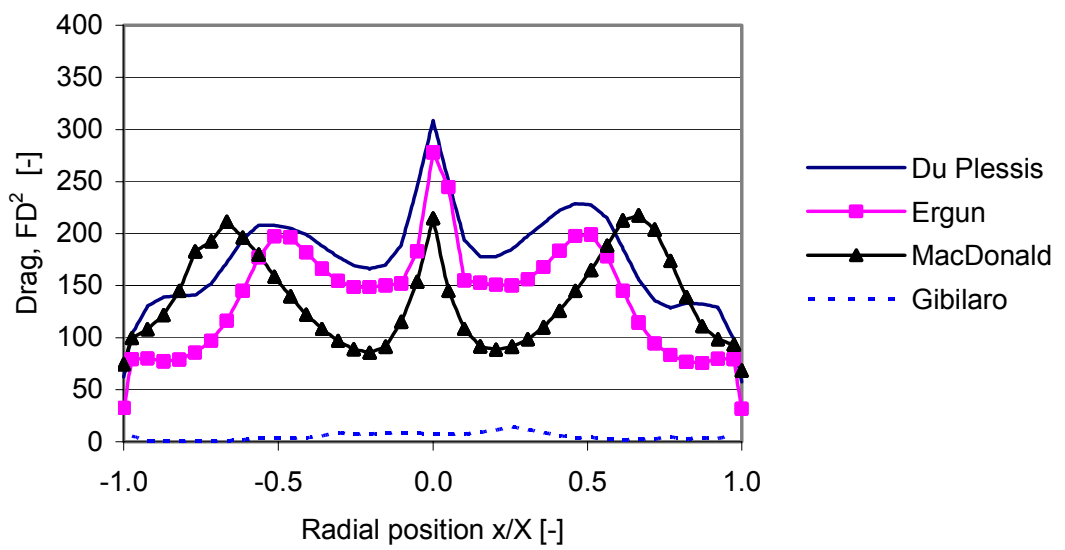

Figure 4: Drag (FD2) as a function of radial position at height $0.3 \mathrm{~m}$ in the bed. 


\section{Discussion}

Different drag models were investigated and their predictions for bubble behaviour in a fluidized bed compared with experimental measurements. Although the overall trends are the same there are some particular discrepancies among the models and further careful investigations are needed for conclusive statements. It seems, however, that the model of Du Plessis and Woudberg is the most promising, since it involves no empirical coefficients and, based on the physical conditions in the bed, adaptations towards improvement could be made in a structured manner. Another positive point of this model is that the same model is used over the whole range of voidages and Reynolds numbers found in a bed.

In simulation of dense particle systems it is important to avoid unphysical high packing. It was observed that the Gibilaro drag model gave a too low bed expansion and too high particle concentrations in parts of the bed. The Du Plessis and Woudberg model gave the highest bed expansion and this might give a more symmetric bed and continuous bubble formation over time. This will be studied in further work.

\section{References}

[1] Yasuna, J.A., Moyer, H.R., Elliott, S., Sinclair, J.L., Quantitative predictions of gas-particle flow in vertical pipe with particle-particle interactions, Powder Technology 84, pp. 23-34, 1995.

[2] Halvorsen, B., Mathiesen, V., CFD Modelling and simulation of a labscale Fluidised Bed, Modeling, Identification and Control, 23(2), pp. 117-133, 2002.

[3] Ibsen, C.H., An experimental and Computational Study of Gas- Particle Flow in Fluidised Reactors, Ph.D. Thesis, Aalborg University, Esbjerg, 2002.

[4] Bokkers, G.A., van Sint Annaland, M., Kuipers, J.A.M., Mixing and segregation in a bidiperse gas-solid fluidised bed: a numerical and experimental study, Powder Technology, 140, pp. 176-186, 2004.

[5] Ergun, S., Fluid flow through packed columns, Chemical Engineering Progress, 48(2), pp. 89-94, 1952.

[6] Bird, R.B., Stewart, W.E \& Lightfoot, E.N., Transport Phenomena, John Wiley and Sons, New York, 1960.

[7] MacDonald, I.F., El-Sayed, M.S., Mow, K. \& Dullien, F.A.L., Flow through porous media - the Ergun equation revisited, Ind. Eng. Chem. Fundam. 18(3), 199-208, 1979.

[8] Gidaspow, D., Muliphase Flow and Fluidization, Academic Press, Boston, 1994.

[9] Rowe, P.N., Drag Forces in a Hydraulic Model of Fluidized Bed- PartII, Trans. Instn. Chem., 39, pp. 175-180, 1961.

[10] Wen, C.Y. \& Yu, Y.H., Mechanics of Fluidization, Chemical Engineering Progress 62, pp. 100-111, 1966. 
[11] Gibilaro, L.G., Di Felici, R. \& Waldram, S.P., Generalized friction factor and drag coefficient for fluid-particle interaction, Chemical Engineering Science 40(10), pp. 1817-1823, 1958.

[12] Du Plessis, J.P., Analytical quantification of coefficients in the Ergun equation for fluid friction in a packed bed, Transport in Porous Media 16, pp. 189-207, 1994.

[13] Woudberg, S., Du Plessis, J.P. \& Smit, G.J.F., On the hydrodynamic permeability of granular porous media, Proc. Int. Conf. on Environmental Fluid Mechanics ICEFM'05, IIT Guwahati, Assam, India, March 2005, pp. 277-283, 2005.

[14] Hasimoto, H., On the Periodic Fundamental Solutions of Fluids Relative to Beds of Spherical Particles, A.I.Ch.E. Journal 4(2), pp. 197-201, 1958.

[15] Churchill, S.W. \& Usagi, R., General expression for the correlation of rates of transfer and other phenomena, A.I.Ch.E. Journal 18(6), pp. 1121$1128,1972$.

[16] Woudberg, S., Flow through isotropic granular porous media, MScEng Thesis, University of Stellenbosch, in progress, 2006.

[17] Geldart, D., Gas Fluidization Technology, John Wiley \& Sons Ltd, 1986.

[18] Mathiesen, V, Solberg, T, Hjertager, B.H., Prediction of gas/particle flow including a realistic particle size distribution. Powder Technology 112, pp. 34-45, 2000.

[19] Halvorsen, B., An experimental and computational study of flow behaviour in bubbling fluidized beds., Doctoral Thesis at NTNU: 70, 2005.

[20] Deardorff, J.W., On the Magnitude of Subgrid Scale Eddy Coefficient. Journal of Computational Physics, Vol. 7, pp. 120-133, 1971. 